\title{
Doppler and proper motions accompanying formation of an additional magnetic flux in the mature solar active region (NOAA 7216)
}

\author{
V. Bumba ${ }^{1}$, M. Klvaňa ${ }^{1}$ and B. Kálmán ${ }^{2}$ \\ 1 Astronomical Institute, Academy of Sciences of the Czech Republic, 25165 Ondrejov, Czech Republic \\ 2 Heliophysical Observatory of the Hungarian Academy of Sciences, H-4010 Debrecen, P.O. Box 30, Hungary
}

Received October 31; accepted December 18, 1995

\begin{abstract}
We document the evolution of an additional magnetic flux in the photosphere of a fully developed sunspot group, we measure longitudinal magnetic field, line-of-sight motions, and proper motions of sunspots in the group during this process. We demonstrate the close correlation of magnetic field, Doppler and proper motion singularities with the area in which additional magnetic flux appears and the new penumbrae and umbrae develop. We discuss the probable reasons for this fact and for the region's weak flare activity. There exist in the group about eight sunspots with the Evershed effect, differing in form, in dependence on the history of development of the spot in which it is observed.
\end{abstract}

Key words: Sun: sunspots - Sun: magnetic field - Sun: doppler motions

\section{Introduction}

In skimming over our magnetic and Doppler velocity maps of solar active regions, we noticed that the growth, renewal or decrease of spot-forming activity in active regions is locally related to the singularities of their magnetic field topologies (inclusions of opposite polarities, or just the boundary of both polarities, usually with large magnetic gradients) which develop very rapidly, as we have already demonstrated earlier (Bumba 1988). These magnetic singularities are accompanied by a specific situation in the distribution of line-of-sight motions, demonstrating that also the velocity field is strongly disturbed, or rather very active, in such locations. We also succeeded in demonstrating that these regions coincide with the knots or sources of proper motions of sunspot nuclei (Bumba et al. 1993; 1995). But the following questions remained unanswered: What are the physics of the relations between these magnetic and velocity field singularities; What is primary, the motions, the magnetic fields, or something else; to what degree does the process depend on the magnitude of the impulse causing the changes, and how to estimate this magnitude?

In continuing our efforts to investigate the mentioned problems, and, generally, to obtain a better understanding of the three-dimensional structure of solar photospheric

Send offprint requests to: V. Bumba motions, the observing logs of the Ondřejov and Debrecen Observatories were compared to find long series of observations of sufficiently complex and developing solar active regions. From the material of the years 1992-94 several active regions were selected, of these NOAA 7216 is studied in the present paper.

This fairly large (approximately 1000 millionth of the solar hemisphere on July 1) active region was seen between June 29 and July 11, 1992, at Carrington coordinates $L=$ $120^{\circ} B=15^{\circ}$. It was fully developed, but demonstrated a probable renewal of activity in the form of a delta magnetic configuration (according to Solar-Geophysical Data SGD, Boulder, CO, U.S.A.) just in the process of formation. It did not produce large flares. Although during its transit across the solar disk a new magnetic flux in the form of bipolar pairs of sunspots emerged in the middle of the old group, and one of these showed an unusual southward proper motion, the largest flare was only of importance 1 in $\mathrm{H}$-alpha. Briefly, it displayed a medium impulse of new magnetic flux production. As a series of magnetograms on July $1-4,8$ and 9 , and daily photographic observations of the photosphere were available, we studied the magnetic fields, line-of-sight (LOS) and proper motions in this active region in their mutual relations. 


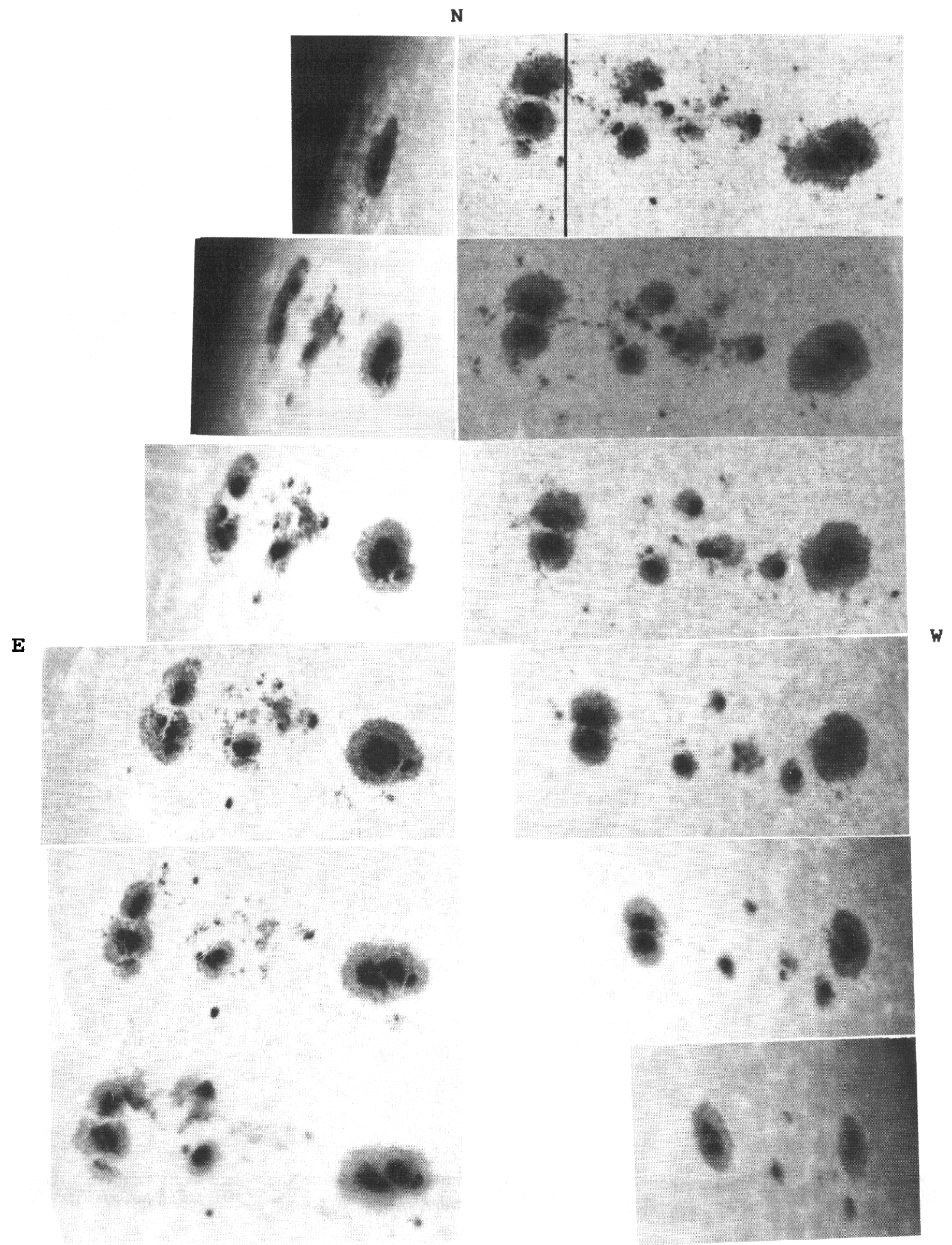

Fig. 1. Series of photographs of NOAA 7216 taken in Debreccn: June 29, 06:45:36 UT; June 30, 07:05:28 UT; July 1, 07:14:48 UT; July 2, 07:55;23 UT; July 3, 08:03:30 UT; July 4, 08:01:04 UT; July 5, 08:23:01 UT; July 6, 07:34:46 UT; July 7, 07:17:26 UT; July 8, 05:35:28 UT; July 9, 05:10:58 UT; July 10, 06:18:08 UT 


\section{Observational data}

\subsection{Photographic observations of sunspots and data reduction}

Whole-disk white-light photographic observations were made with the photoheliograph of the Debrecen Heliophysical Observatory (Fig. 1), for details see Bumba et al. (1993). In the period June 29-July 10, 1992, the following number of plates were measured (in format monthday/number of plates): $6-29 / 3,6-30 / 3,7-01 / 9,7-02 / 3,7-$ $03 / 11,7-04 / 3,7-05 / 4,7-06 / 3,7-07 / 3,7-08 / 3,7-09 / 2$ and $7-10 / 3$. Although the number of photographic observations was limited for economical reasons, the active region did not show any very rapid evolution, so that most of the umbrae could be reliably identified from one day to the next, their coordinates measured on every plate. In the reductions of the measurements from rectangular to solar coordinates, corrections were applied in respect of the position angle zero error, the plate tilt, the distortion of the enlarging lens of the photoheliograph, and of differential atmospheric refraction (Kalman 1980). The individual measurement results were then averaged using a cubic spline fit. Besides this, the contours of the penumbrae and umbrae of the best observation of every day were measured, approximately 1500 points per plate, to produce sunspot drawings free of geometric projection effects.

\subsection{Photoelectric observations}

Photoelectric observations of the longitudinal magnetic fields, line-of-sight velocities with enhanced sensitivity and intensity in continuum and in the line used (mostly FeI $(\lambda)=5253.47(\AA)$ were made at the Ondrejov observatory (Klvaña \& Bumba 1994a, b). The series of observations started on July 1,1992, but was interrupted because of weather on July $5-7$, to continue again during the two next days (see Table 1). All measurements were made in the normal $(\mathrm{N})$ scanning mode with a spatial resolution of $4.8^{\prime \prime} \times 3.2^{\prime \prime}$.

\section{Magnetic field and sunspot development}

\subsection{Longterm evolution of activity in the area}

It seems that there were no regular cells of the background field around $L=120^{\circ}, B=15^{\circ}$ two rotations earlier (No. 1855). One rotation earlier (No. 1856) a complex multipolar structure was observed on the synoptic magnetogram. It produced at least two active regions (NOAAs 7186 and 7191). The former seemed to be fully developed already at the time it appeared on the disk, declining steadily thereafter, while the second one displayed its evolution on the visible disk, its growth being apparently connected with the decay of the former, even creating a small satellite group (NOAA 7193) northwards of it, towards its end.
During the next rotation after the appearance of the studied group (No. 1858), magnetic fields of both polarities dispersed widely, but both main spots remained in the leading, as well as in the following part (NOAAs 7241, 7242). Two rotations later a still larger bipolar feature, but without larger concentrations, was observed, the line dividing polarities running approximately through the area, where the largest motions in the studied NOAA 7216 (umbrae 2, 3, 4 an the $\mathrm{K}$ complex) were observed.

\subsection{Sunspot group observed in white light}

AR NOAA 7216 appeared to be a fairly usual large bipolar region as it emerged at the eastern limb on June 29, 1992 (Figs. 1-3). At the preceding (western) end it had a large triple-umbra $(10,11$ and 12$)$ in the compact, regular leading spot, at the eastern end a more dispersed following spot (umbrae A, BA, BB, BC and BD in a common penumbra). In the middle there was a disturbed part with an irregular penumbra and umbrae of both polarities, this was the delta area. The most stable f-polarity umbrae were medium-sized $\mathrm{M}$, and the small spot $\mathrm{N}$ at the southern border of the group in this area. In the northern part of the irregular penumbra small, short-lived umbrae of p-polarity 2,21,22 moved westward, of these umbra 2 fairly quickly and steadily (Figs. 3, 4).

A new development began on July 3 , when a bipolar spot pair appeared in the vicinity of umbra $A$, in the following part. Its leader was 4 , followers were ZA and ZB. They grew quickly, 4 rushed forward (to the west), ZA and $\mathrm{ZB}$ converged and moved towards $\mathrm{A}$, they coalesced to July 5 and formed $\mathrm{Z}$, this in turn attached to $\mathrm{A}$. The emergence of this new magnetic flux was probably the cause of the only Imp. 1 flare in the region on July 3 .

Another new dipole emerged on July 5 in the area between the leader spot and $\mathrm{M}$. The mentioned spots were moving apart, 10,11, 12 westward, $M$ eastward and the irregular penumbra in the middle of the group also broke up by July 4 , as decaying umbra 2 moved westward nearer to the leader spot. The new dipole consisted of the $\mathrm{p}$ polarity spot 3 and f-polarity spots KA, KB (later also $\mathrm{KC}, \mathrm{KD}$ ). What was remarkable, was that these oppositepolarity umbrae did not diverge, as usual, but converged, and moved together to the south (Figs. 3,4). Umbra 3 moved almost exactly due south 3 heliographic degrees in 5 days. The interaction of these spots was distinct in their penumbral structure. They had strongly asymmetric penumbrae, elongated towards the opposite polarity pair (Figs. 1-3). This tendency, to a lesser extent, was also visible in the case of the bipolar pair 4-Z.

\subsection{Active regions' magnetic field development}

The first photoelectric measurements of the investigated region were obtained at Ondrejov with the aid of the magnetograph on July 1, 1992 at about 12:15 UT. From the 
Table 1. Photoelectric measurements of NOAA 7216 at Ondřejov

$\begin{array}{lcccl}\text { Date } & \text { File number } & \text { Scanning mode } & \text { Time of obser. (UT) } & \text { Notes } \\ \text { July 1 } & 070192-1 & \mathrm{~N} & 12: 15: 13-12: 26: 11 & \\ \text { July 2 } & 070292-1 & \mathrm{~N} & 08: 05: 16-08: 16: 54 & \\ & 070292-3 & \mathrm{~N} & 11: 38: 10-11: 57: 23 & \\ \text { July 3 } & 070292-4 & \mathrm{~N} & 13: 39: 23-14: 19: 22 & \\ & 070392-1 & \mathrm{~N} & 07: 33: 04-07: 47: 52 & \\ & 070392-3 & \mathrm{~N} & 08: 40: 18-09: 29: 31 & \text { clouds } \\ \text { July 4 } & 070392-4 & \mathrm{~N} & 12: 31: 19-12: 49: 02 & \text { clouds } \\ & 070392-5 & \mathrm{~N} & 12: 59: 28-13: 31: 03 & \text { not finished } \\ \text { July } 8 & 070492-1 & \mathrm{~N} & 10: 06: 16-10: 33: 14 & \text { clouds } \\ & 070492-2 & \mathrm{~N} & 11: 23: 03-11: 59: 51 & \\ & 070892-1 & \mathrm{~N} & 08: 15: 14-08: 25: 33 & \\ & 070892-2 & \mathrm{~N} & 08: 41: 49-08: 52: 14 & 5123.73 ; g=0 \\ & 070892-3 & \mathrm{~N} & 09: 03: 43-09: 15: 38 & \\ & 070892-4 & \mathrm{~N} & 09: 28: 16-09: 38: 22 & \\ \text { July 9 } & 070892-5 & \mathrm{~N} & 09: 47: 41-09: 58: 53 & 5250.65 \\ & 070892-6 & \mathrm{~N} & 10: 58 ; 56-11: 11: 48 & 5123.73 ; g=0 \\ & 070892-7 & \mathrm{~N} & 11: 23: 55-11: 35: 59 & 5123.72 ; g=0 \\ & 070892-12 & \mathrm{~N} & 14: 18: 39-14: 30: 19 & \\ & 080992-5 & \mathrm{~N} & 08: 26: 49-08: 40: 41 & \end{array}$

topology of the regions' magnetic field it is clear at first glance (Figs. 5 and 6) that this field is not new, both of its polarities being extended far souhtwards. But at the same time, it formed powerful concentrations, in the leading, negative polarity spot, stronger than usual, with three nuclei (see the preceding paragraph - in our magnetic maps only two concentrations were mostly seen, the inclination of their axis changing continuosly), as well as in the positive polarity, even more complicated following spot, with many more continuously coalescent nuclei. These two main concentrations could be observed even during the next solar rotation (they were indicated as two NOAAs (Nos. 7241 and 7242, see also Fig. 5). At the same time the remainders of both polarities were extended in two, almost parallel, long strips, stretched to the south-west.

During the first day of our photoelectric observations, the following part of the region displayed another two three positive polarity concentrations, evolving in the central part of the group, not far from the main boundary between both polarities. At the beginning, this evidently new positive polarity magnetic flux seemed to be much more developed than its negative polarity counterpart, situated north-west of it. The positive magnetic flux in this central part of the region highly exceeded the negative polarity flux during the whole of the first four days of measurement. Only the last day of the photoelectric observations were both fluxes nearly balanced (Fig. 6).
In the white light, this is the disturbed part of the group with irregular penumbra and umbrae of both polarities. The most stable spot $M$ represented the main positive polarity maximum, which developed first and was observable till the end of our photoelectric observations, while spot $\mathrm{N}$ represented a small secondary concentration, observed best on the magnetic maps of July 3, while on the next day, only a small remnant of it was visible.

A new negative flux developed at the beginning in small islands, adjacent to positive polarity, but without large magnetic gradients. Each such island seemed to have attached (within a relatively short time interval) a corresponding positive polarity maximum, with a longer life time than the negative polarity feature. It appears that both maxima are formed by one physical process in which the growths of both polarities mutually induce themselves. In this way the pairs of nuclei $Z, 2+4$ and $K, 3$ developed. And, of course, the new magnetic flux activity precedes the formation of the nuclei.

During the last day of the photoelectric observations, the concentrations of opposite polarities were clearly separated. From the central part of the new activity only nuclei $\mathrm{M}, \mathrm{K}$ and 4, 3 remained, nucleus 3 became attached to the same negative polarity leading spot. Without white-light observations we would say that nucleus 3 represents one of the leading spot nuclei, separated from the main spot Fig. 6). 


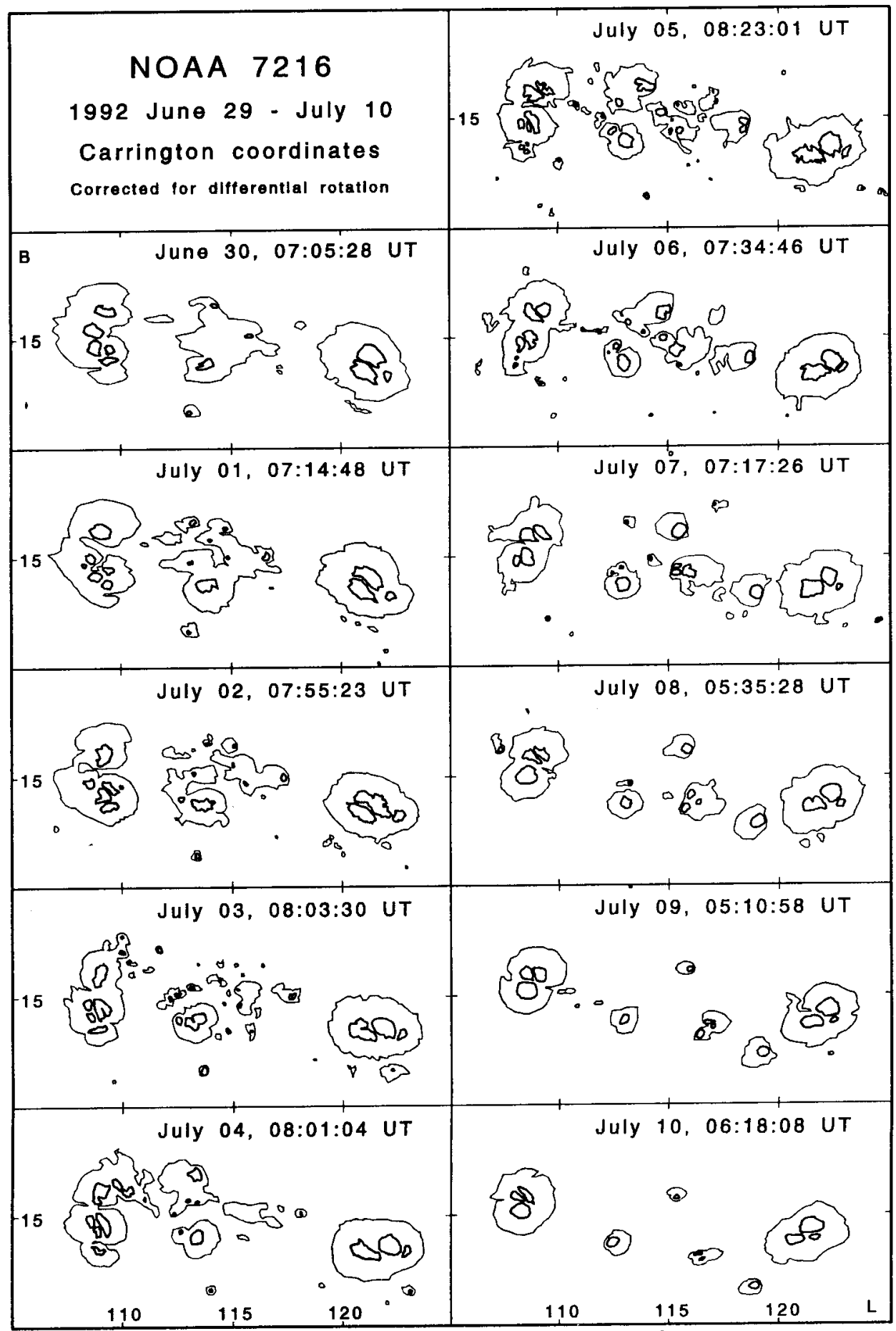

Fig. 2. Development of NOAA 7216 displayed in Carrington coordinates, corrected for differential rotation

\section{Motions in the group}

\subsection{Character of the individual proper spot motions}

Trajectories of the longer-lived umbrae from spline fits are shown in Fig. 4, where the arrowheads denote the positions at 12:00 UT every day. The first and last days of observation for every labeled umbra are given in Table 2 . According to their motion, the umbrae can be divided into six different clusters (Fig. 7).

Clusters 10 and $\mathrm{ABC}$ at the preceding and following ends of the active region represent the usual motions found in mature bipolar sunspot groups. Cluster 10 contains the 
NOAA 7216

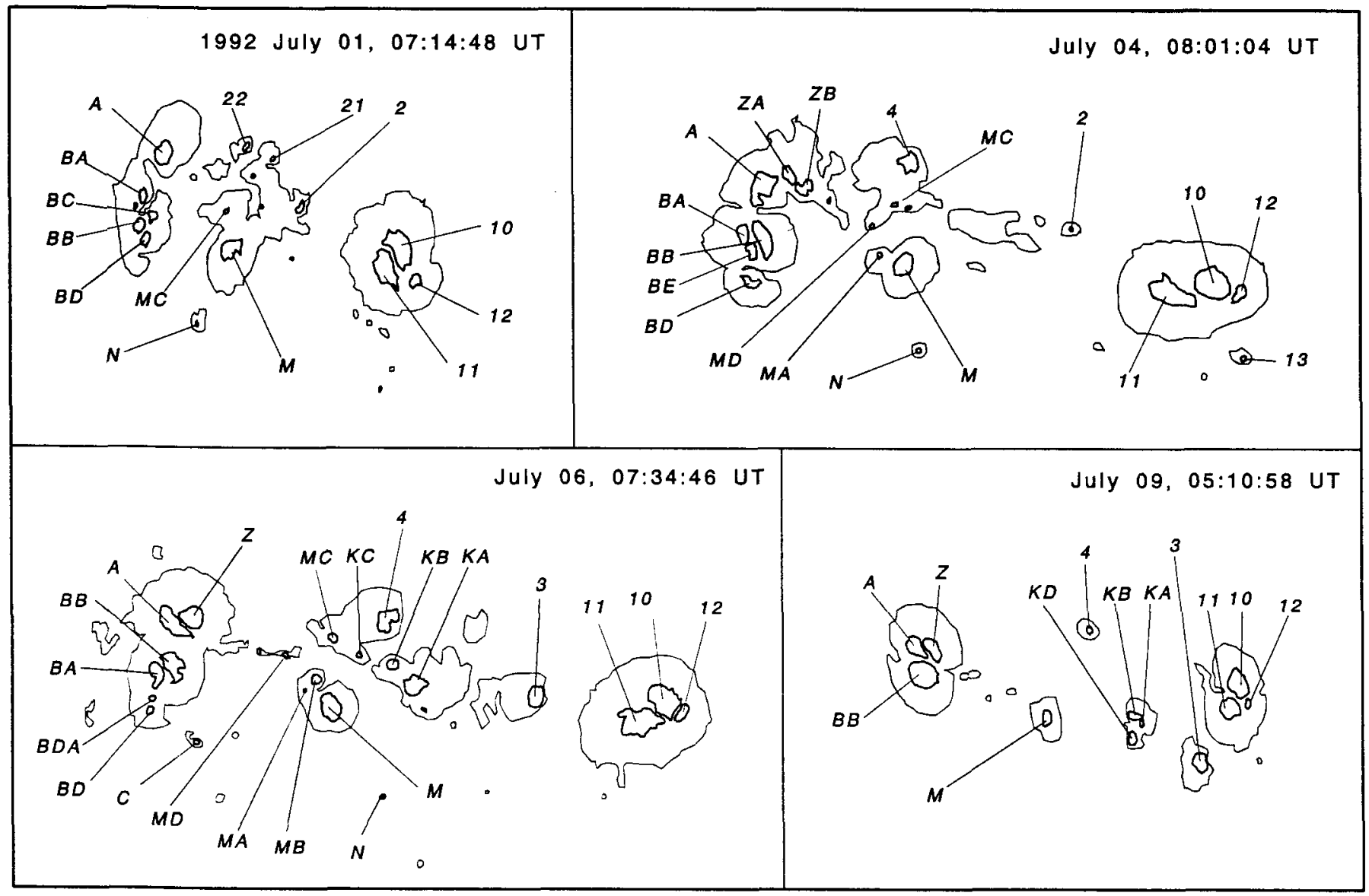

Fig. 3. Drawings of NOAA 7216 from penumbra and umbra contour measurements, with designations of the longer-lived umbrae. P-polarity (negative) umbrae are labeled with numbers, f-polarity (positive) with letters

multiple umbrae of the leader spot $(10,11,12)$ and a small p-polarity satellite spot 13 . The latter moves forward (to the west), as is typical for p-spots. The umbrae in the large leader spot move with a slow counterclockwise circulation; this type of motion of the leader spots has already been described by Waldmeier (1955). Also typical is the chaotic, but generally backward motion of the umbrae in cluster $\mathrm{ABC}$, containing the more dispersed, main follower part and small satellite f-spot $\mathrm{C}$.

In the middle of the group, clusters 10 and $\mathrm{MN}$ move differently, according to their magnetic polarity. Cluster 20 contains relatively small and short-lived p-polarity umbrae, moving forward and with higher velocities (of the order of $100 \mathrm{~m} / \mathrm{s}$ ) than the f-polarity umbrae in cluster $\mathrm{MN}$. The latter move chaotically and their velocity is smaller by one order of magnitude. These motions of the main spots also demonstrate the stage of the full growth of the group, as the magnetic fields did. This is best seen in Fig. 8 , which shows the proper motions of spots related to leading nucleus 10 .
The emerging new bipolar spot pairs in clusters 4-Z and $3-\mathrm{K}$ move very differently, depending on their position relative to the old spotgroup. Cluster 4-Z emerges on July 3 in the northeastern part of the active region and can develop freely, as small umbrae 21, 22, ahead of it, disappeared at this time. The vigorously growing umbra 4 can thus move forward quickly (in the first three days with a velocity of around $200 \mathrm{~m} / \mathrm{s}$ ). Later it regularizes and slows down, it begins to decay, and on the last day (by July 10) it even turns back. The f-part of the dipole (ZA-ZB) seems to be attracted to the old follower umbra $A$, and after July 5 they move together slowly eastward.

The most unusual motions are in cluster $3-\mathrm{K}$. This dipole appears on July 5 and its p-polarity spot 3 moves with an almost constant $80 \mathrm{~m} / \mathrm{s}$ velocity to the south. This spot becomes quickly regular, already by its second day, and its penumbra is elongated towards $\mathrm{f}-\mathrm{spots} \mathrm{K}$, which indicates a close magnetic connection between them. These f-polarity umbrae also move unusually, convergently and to the southwest, they also have a high velocity of the order of $100 \mathrm{~m} / \mathrm{s}$. Notice in Figs. 1 and 2 for July 7 that 


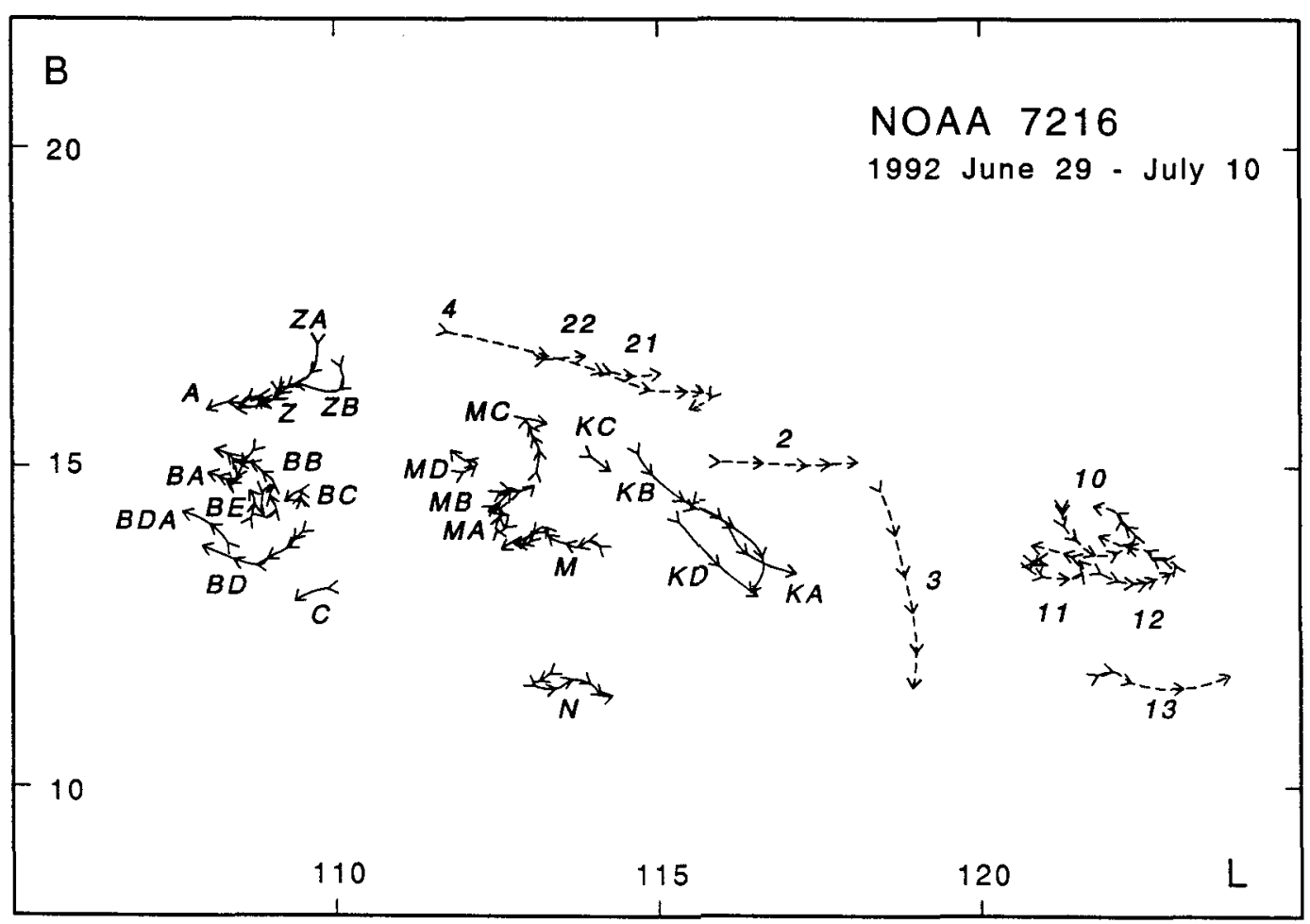

Fig. 4. Trajectories of umbrae in NOAA 7216 from spline fits to the coordinate measurements. Arrowheads give the positions at 12:00 UT every day. Dashed line and numbers denote p-polarity, full line and letters - f-polarity. Carrington coordinates corrected for differential rotation. Labels of umbrae can be found in Fig. 3, first and last days of their observation in Table 2

f-polarity umbrae KA, KB, KD are twice as close to ppolarity umbra 4 than to p-polarity umbra 3 of similar size, but their penumbra is elongated towards 3 , indicating a genetic connection between them, as this is also visible in their common motion.

\subsection{Line-of-sight velocity measurements}

\subsubsection{Evershed effect}

The most striking features in the morphology of the Doppler velocity distribution maps are at first glance, the patterns of the Evershed effect, attached at the beginning to each fully developed sunspot, and practically to every remaining spot in the group, at the end of the photoelectric observations (Fig. 6). From the point of view of the Evershed effect, these final observations are very instructive: all spots demonstrate the Evershed flow, but the individual flows differ substantially in amplitudes and the ratio of velocities of both motions, following, very probably, the history of spot development. Only spot K, which was very close to dissapearance, is practically without positive (toward the observer) motions. We remind the reader that on July 8 some measurements were made in the line without split (see Table 1 and Bumba \& Klvaña 1995). We will investigate these different behaviors of the Evershed flow patterns in more detail elswhere.

\subsubsection{Doppler motions connected with the development} of new magnetic fluxes

The most interesting distribution of the line-of-sight velocities can be seen in the velocity maps of July 1 to July 4 in the central part of the group, in the area where the new magnetic flux formed and the new penumbra and nuclei apeared (again Fig. 6). Spot M already seems to have its Evershed flow patterns, but north-west of it, where the new magnetic flux is developing, we see a motion pair (relatively large velocities of more than $800 \mathrm{~m} \mathrm{~s}^{-1}$ in both directions) the boundary of which is perpendicular, and on July 2 partly, on July 3 even opposite to that of the Evershed motions. This concerns pairs Z - 4 on July 1, as well as K -2 on July 2 . On July 3 just the cluster of nuclei $\mathrm{K}$ has the orientation of both motion polarities opposite to that of the Evershed effect, and on July 4 practically the whole area of newly formed penumbra and nuclei has the negative velocity sign (away from the observer), with a small exception in the center of the cluster of nuclei $\mathrm{K}$, where an island of small positive velocities exists. On July 8 , the motions with negative velocities occupy practically the whole region with small islands of positive motions in the Evershed flow patterns of each sunspot. 


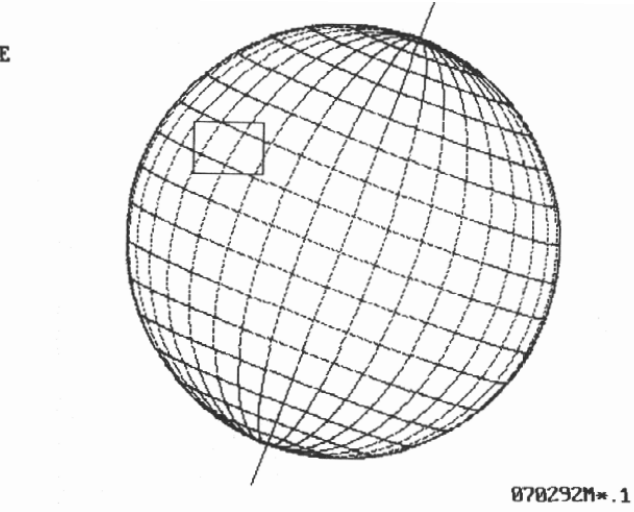

s

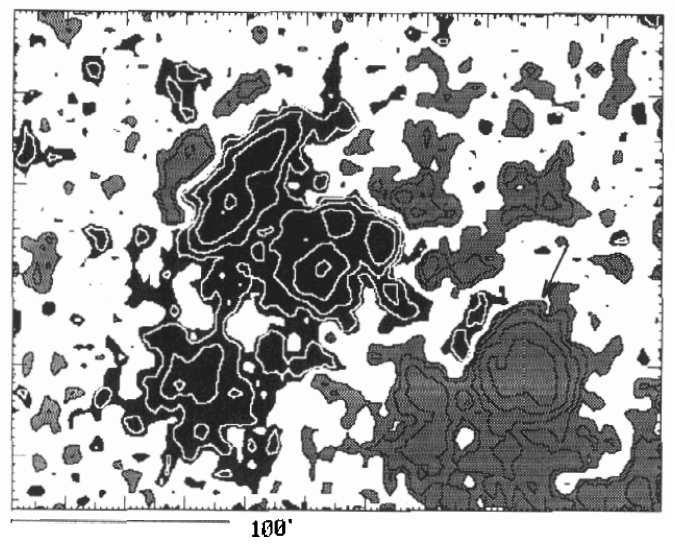

7216

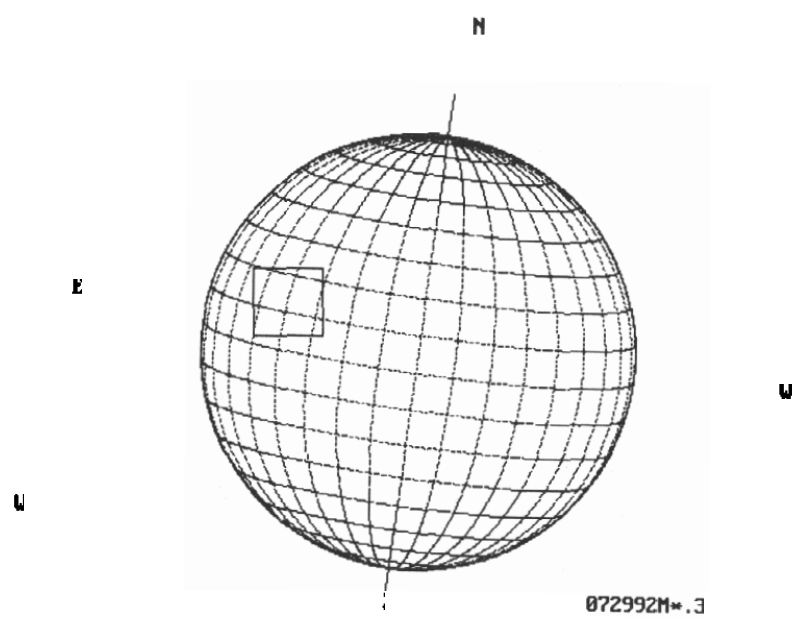

$\mathbf{s}$

$7242 \quad 7241$

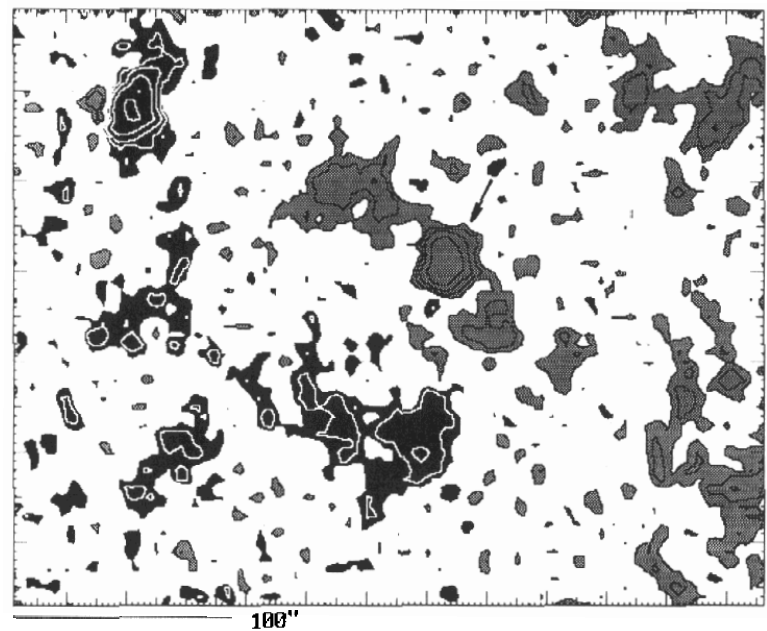

Fig. 5. Magnetic charts of NOAA 7216 on July 1, 1992 at 12:15:13-12:26:11 UT (left hand side) and of NOAAs 7241 and 7242 on July 29, 1992 at 13:16:57-13:34:00 UT one rotation and one day later (right-hand side). The first isogauss lines indicate 40 Gauss of the longitudinal magnetic field component, the intensity scale is logarithmic with coefficient 2 . Positive polarity is darker. The leading spots are indicated by arrows

\section{Discussion of results}

\subsection{The stage of the new magnetic flux formation in} NOAA 7216

If we summarize the observations demonstrated above, we may conclude that, in the case of the obscrved active region NOAA 7216, two newly emerging dipoles interacted within an old, large bipolar surspot group. At the same time this impulse of the new magnetic and photospheric activity is not too large. The amount of the newly added magnetic flux amounts to about $610^{21} \mathrm{Mx}(60 \mathrm{TWb})$, about $15 \%$ of the whole region's magnetic flux $\left(410^{22}\right.$ $\mathrm{Mx}$, or $400 \mathrm{TWb}$ ). The new flux emerges more probably in developed active regions (Liggett \& Zirin 1985), and the new spots can be distinguished from the old ones according to the character of their motion (Kalman 1980); interaction between the new and old spots can also take place. In our case new dipole 4-Z developed almost unhindered and showed the usual divergent motion of new spotgroups, p-polarity (umbra 4) moving quickly forward, f-polarity (ZA, ZB, Z) more slowly backward. The other new dipole, 3-K, was blocked on two sides by the leader spot, and the middle MN cluster was pushed from the north by the quickly moving and evolving spot 4 , so that the umbrae of the $3-\mathrm{K}$ dipole moved southward, the f-spots even southwestward, in the only unblocked direction. The 

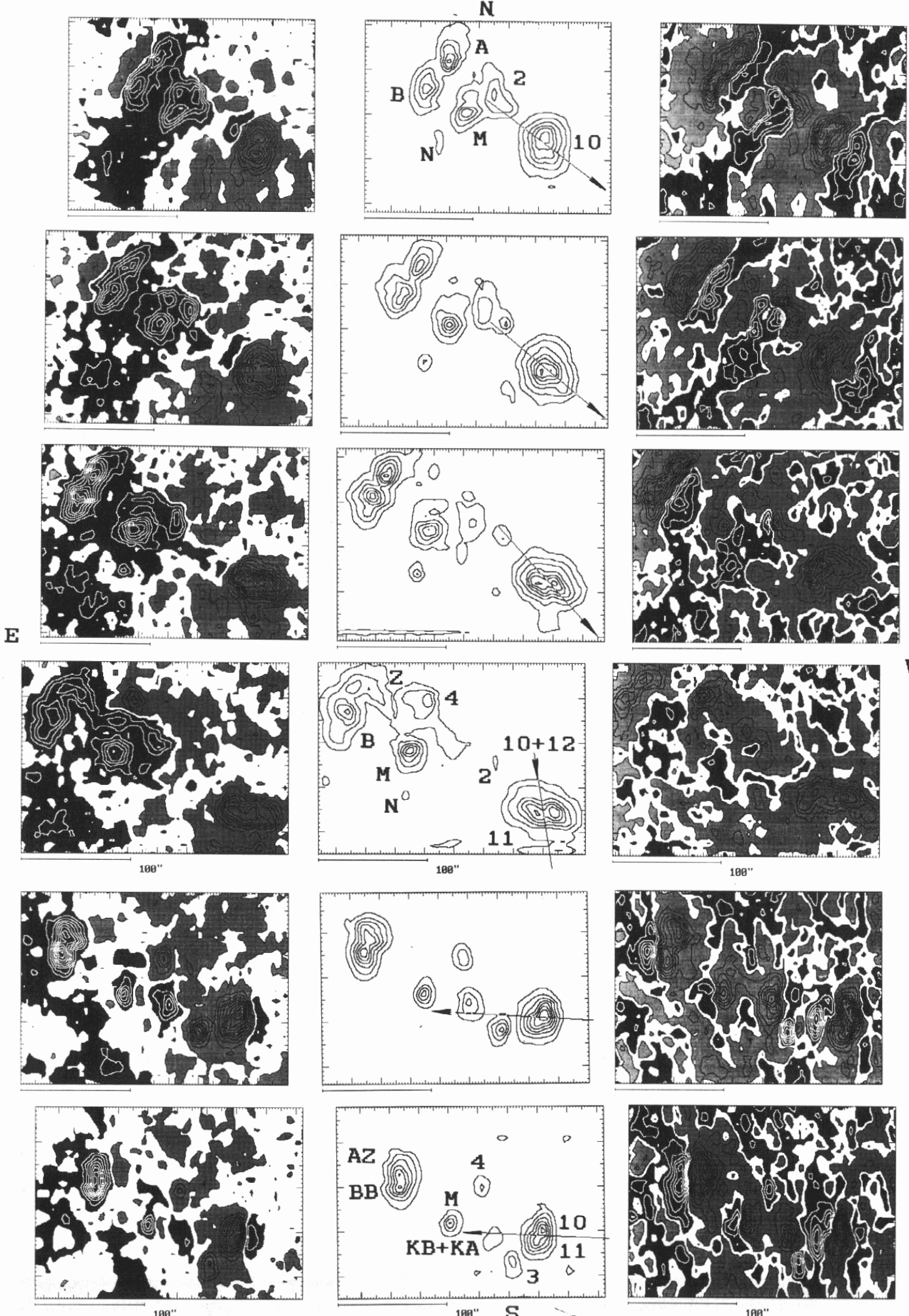

Fig. 6. Series of maps of the longitudinal component of the magnetic field (to the left), continuum intensity (in the middle), and of the line-of-sight velocity component distribution (to the right) for six days of magnetographic observations of the sunspot group NOAA 7216: July 1, 12:15:13-12:26;11 UT; July 2, 08:05:16-08:16:54 UT; July 3, 07:33:04-07:47:52 UT; July 4, 10:06:16-10:33:14 UT; July 8, 08:15:14-08:25:33 UT; July 9, 08:26:49-08:40:41 UT. The first isogauss lines represent an intensity of 40 Gauss, the scale is linear, lines in steps of 120 Gauss. The positive polarity is darker. On the continuum intensity maps the direction toward the disk center is indicated by an arrow going through the center of gravity of the leading spot. Individual spots or their clusters are indicated by labels as in Fig. 3. On the Doppler velocity maps the first velocity lines are drawn for $50 \mathrm{~m} \mathrm{~s}^{-1}$, the subsequent lines in steps of $200 \mathrm{~m} \mathrm{~s}^{-1}$. The areas of positive motions (toward the observer) are darker 


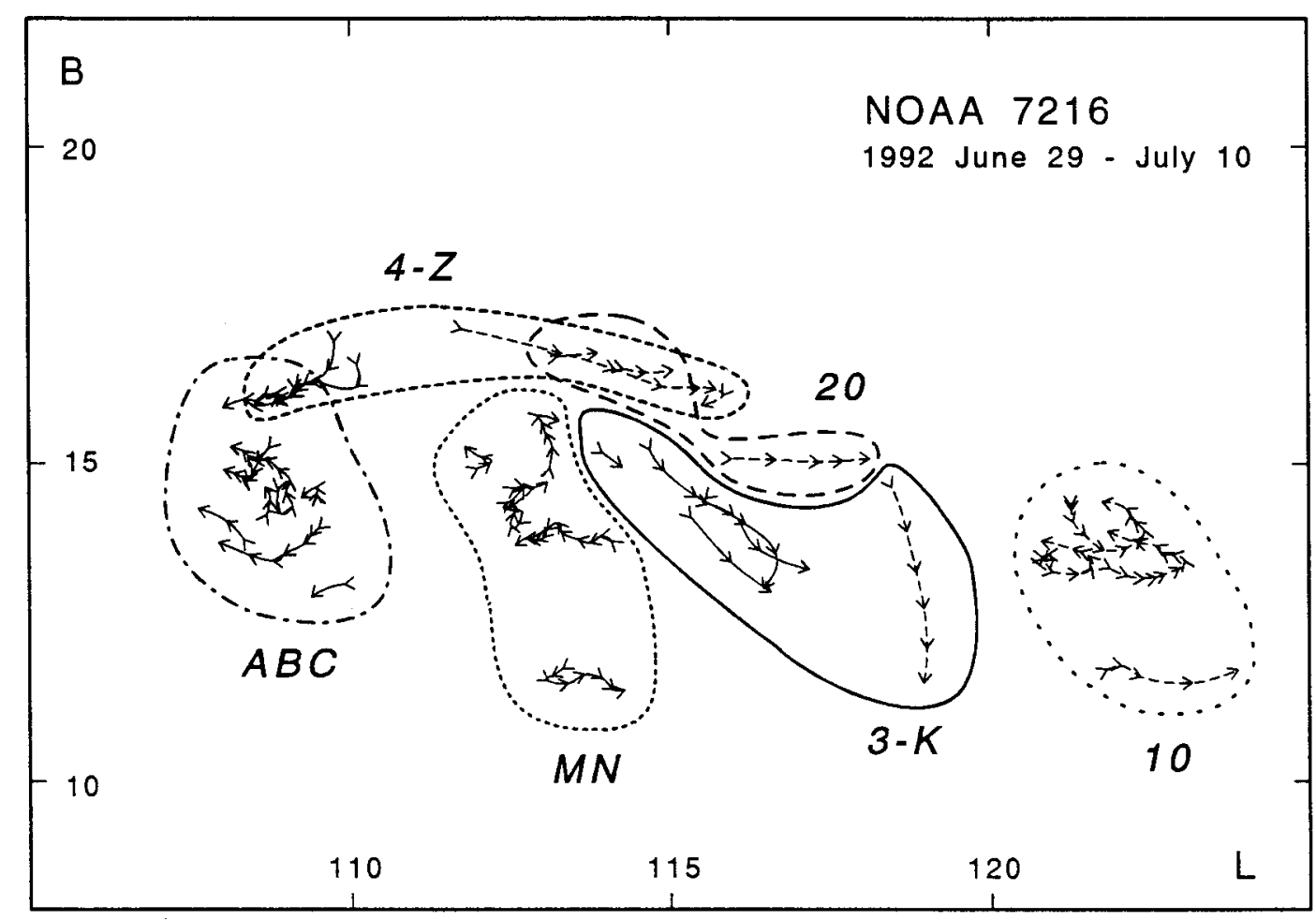

Fig. 7. The six clusters of umbrae which can be determined from the character of their proper motion. For detailed description refer to text

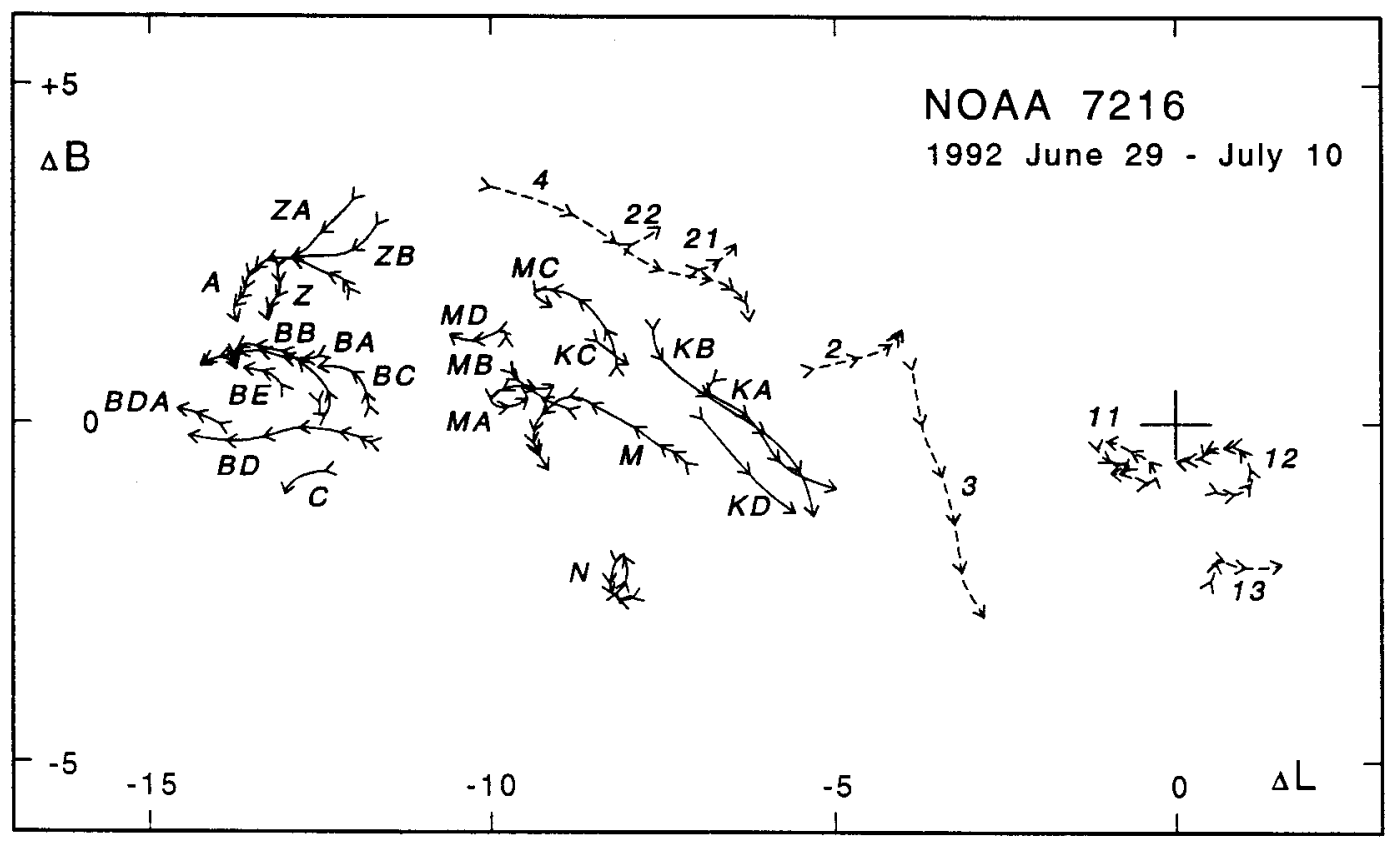

Fig. 8. As in Fig. 4. Only the proper motions of individual spots are related to the nucleus of the leading spot 10 
umbrae in this dipole maintained their connection, and did not interact with the surroundings.

\subsection{New activity is related to both the magnetic and motion field singularities}

As we have demonstrated earlier (Bumba 1988; Bumba et al. 1993 and 1995) the new magnetic flux develops in the place, where at least opposite polarity magnetic fields make contact and certain magnetic gradient exists. The magnetic situation can be more complicated, gradients large, the developing activity is then also very large. In the same area we observe large Doppler motions, usually of both directions, and the place serves as the radiant of proper spot motions, or the proper motions have their knots in this area.

The same seems to be true in the case of the additional activity of NOAA 7216, only the new magnetic flux is of medium value, and it is produced during two-three days only. Therefore, the photospheric, and as we will see, also the chromospheric activities are not very large.

The comparison of our magnetic and line-of-sight velocity maps indicates that in the most active area firstly large motions are observed, followed by magnetic flux growth, later photosheric activity appears. And the time lags seem to be of the order of 10-20 hours. But confirm this physically very important result with a higher precision, the development of several more actitive regions, whose activity impulses at various stages of their development are of different intensity, have to be studied. Nevertheless, studying the diminishing leading complex of NOAA 6850 , we have found that this time-lag was of an opposite sign; the Doppler motion anomaly disappeared before the opposite magnetic polarity inclusion to which it was bound (Bumba et al. 1995).

Thus, the above demonstrated facts about the close correlation of the new magnetic flux and sunspot development with occurrence of large line-of-sight velocities and radiants of new proper spot motions, and the mutual time dependence of magnetic and Doppler motion changes must indicate that the motion of photospheric plasma has to be involved in the proper process of magnetic field generation, its strenghtening or weakening. To decide this problem of the nature of the physical process of local magnetic field development, or in other words, about the reality of the possible existence of a "local dynamo", more observational data, concerning, above all, the development of ordinary active regions, are required.

\subsection{Flaring activity}

The flare activity of this active region was small, according to Solar-Geophysical Data on the average 5-8 subflares per day between June 30 and July 6, 2 subflares per day before and after this period. The largest flare was an Imp. 1 in $\mathrm{H}$-alpha on July 3 , this was probably connected with
Table 2. Umbrae in NOAA 7216

\begin{tabular}{|c|c|c|c|}
\hline Umbra & Magn. pol. & First seen & Last seen \\
\hline 10 & - & June 29 & July 10 \\
\hline 11 & - & June 29 & July 10 \\
\hline 12 & - & June 29 & July 10 \\
\hline 13 & - & July 01 & July 05 \\
\hline 2 & - & June 30 & July 04 \\
\hline 21 & - & June 30 & July 02 \\
\hline 22 & - & July 01 & July 02 \\
\hline 3 & - & July 05 & July 10 \\
\hline 4 & - & July 03 & July 10 \\
\hline A & + & June 30 & July 10 \\
\hline $\mathrm{BA}$ & + & June 30 & July 07 \\
\hline $\mathrm{BB}$ & + & June 30 & July 10 \\
\hline $\mathrm{BC}$ & + & June 30 & July 03 \\
\hline $\mathrm{BD}$ & + & June 30 & July 06 \\
\hline $\mathrm{BE}$ & + & July 03 & July 05 \\
\hline $\mathrm{BDA}$ & + & July 05 & July 07 \\
\hline $\mathrm{C}$ & + & July 05 & July 06 \\
\hline KA & + & July 05 & July 09 \\
\hline $\mathrm{KB}$ & + & July 05 & July 10 \\
\hline $\mathrm{KC}$ & + & July 06 & July 07 \\
\hline $\mathrm{KD}$ & + & July 07 & July 09 \\
\hline M & + & June 29 & July 10 \\
\hline MA & + & July 02 & July 08 \\
\hline MB & + & July 05 & July 08 \\
\hline $\mathrm{MC}$ & + & July 01 & July 06 \\
\hline $\mathrm{MD}$ & + & July 03 & July 12 \\
\hline $\mathrm{N}$ & + & June 29 & July 06 \\
\hline $\mathrm{ZA}$ & + & July 03 & July 04 \\
\hline $\mathrm{ZB}$ & + & July 03 & July 04 \\
\hline $\mathrm{Z}$ & + & July 05 & July 10 \\
\hline
\end{tabular}

the emergence of the 4-Z dipole. The magnetic configuration of the active region, although described in SGD as delta between June 30 -July 5, was fairly open, with no large magnetic gradients. The umbrae collided only in the following part between $\mathrm{A}$ and $\mathrm{Z}$, this was slow and between umbrae of the same polarity. Although quick and unusual north-south motions were observed, these were connected with a new dipole (3-K) whose components interacted only with each other and not with the surroundings, as the 1-A pair in Bumba et al. (1995); furthermore the magnetic polarities were "in place", $p$ near $p, f$ near $\mathrm{f}$, even so the spots of this $3-\mathrm{K}$ cluster avoided collision and moved towards the open area. This lack of interactions between umbrae of different polarities belonging to different dipoles, can explain the small flare activity in this region. A similar development of new dipoles inside 
an old, wide bipolar region was described by Gaizauskas et al. (1994), but in that case collision and shearing motion between umbrae of different magnetic polarity led to more activity. Proper motions, leading to large magnetic gradients (Bumba et al. 1993) can lead to flare activity, and shearing motions may increase it.

\section{Conclusions}

The investigated active region represents certain evolutionary stage of a higher unit of solar activity, possibly of a complex of activity, living for several solar rotations.

The observed appearance of the new magnetic flux and the related activity in NOAA 7216 demonstrates the renewal of magnetic activity in an older, relatively large, already fully developed sunspot group. The process lasted for a few days only.

The new magnetic flux developed in two subsequent portions at the inner magnetic field boundary of the region, where the negative leading field bulged northeastward along the north-western part of the positive following field (Figs. 5 and 6). This was also the area of the new penumbra and umbral nuclei formation. The newly developed umbrae diverged fast from this area.

The new magnetic flux was highly unbalanced at the beginning, with a large predominance of positive following flux, and also formed more concentrations than the negative leading flux.

The proper motions of the main spots also demonstrated the stage of the full growth of the group. Their motions were very slow. Consequently the diagrams of trajectories of umbrae in Carrington's coordinates do not differ substantially from those, related to the main leading spot 10 (Figs. 4 and 8 ). The newly developed spots moved faster in the leading field area, the maximum velocity reached a value of about $200 \mathrm{~m} \mathrm{~s}^{-1}$ (umbra 4).

In the area of new magnetic flux and spot development we see Doppler motions in both directions, the velocities of which are larger than $800 \mathrm{~m} \mathrm{~s}^{-1}$, with the mutual orientation of the positive and negative motion areas differing substantially from the orientation of motion pairs in the Evershed effect.
The most important result, the close correlation of areas with new magnetic flux and spot formation with large Doppler motions and radiants of new proper spot motions, and possible time dependence of mutual development of magnetic and Doppler motion fields indicate the involvement of plasma motions in the proper process of, at least, magnetic field strengthening, if not of its generation.

The region's small flare activity may be connected with the lack of interactions between umbrae of different polarities in the region. Even so, the observed flares were closely related to individual specific developments, as to place, and time dependence.

During the whole observational period about eight regular sunspots were observed in the group, each of which demonstrated its own Evershed flow. But all these individual Evershed flow patterns, some of them changing relatively rapidly, differed in mutual amplitude and ratio of velocities of both motion directions in dependence on the life-time and history of the spot development.

Acknowledgements. Observations in Debrecen were made by O. Gerlei, A. Ludmany and I. Nagy. This research was partially supported by grant T-015761 of the Hungarian Scientific Research Fund (OTKA). Bumba and Klvaña would like to acknowledge the support of this work by Grant No. 202/93/0892 of the Grant Agency of the Czech Republic.

\section{References}

Bumba V., 1988, Bull. Astron. Inst. Czechosl. 39, 129

Bumba V., Klvaña M., 1995, Solar Phys. 160, 245

Bumba V., Klvaña M., Kálmán B., Gyori L., 1993, A\&A 276, 193

Bumba V., Klvaňa M., Kálmán B., 1995, A\&AS 109, 355

Gaizauskas V., Harvey K.L., Proulx M., 1994, ApJ 422, 883

Kalman B., 1980, Thesis, Pulkovo Obs.

Klvaña M., Bumba V., 1994a, Handling and Archiving Data from Ground-based Telescopes. In: Albrecht M. and Pasian F. (eds.), ESO Conference and Workshop Proceedings No. 50 (ESO Garching), 172

Klvaňa M., Bumba V., 1994b, JOSO Annual Report 1993. In: Alvensleben A.V. (ed.), 119

Liggett M.A., Zirin H., 1985, Solar Phys. 97, 51

Waldmeier M., 1955, Ergebnisse und Probleme der Sonnenforschung, Akademische Verlagsgesellschaft, Leipzig, p. 170 